\title{
Qualitative and quantitative approaches to highly local behaviors of historical masonry structures
}

\author{
D. Mencías, J. García \& F. Magdalena \\ Technical University of Madrid UPM, Madrid, Spain
}

\begin{abstract}
Many simple methods of analysis for the overall performance of masonry structures can be found; almost all of them are performed to provide safe statements about the stability of this type of structures. However, and quite frequently indeed, problems appear in masonry structures, not globally but at a local level, for which the usual methods do not offer any reliability, especially when the bonding material is damaged and the adherence resistance depends on friction. These local performances represent a practical problem in certain cases, when a proposal of models that correctly approach this local behavior is needed. In this study, we propose a combination of qualitative results of two quantitative methods (both numerical) and an experimental method (which can only indirectly provide quantitative results).This combination will show the nature of the phenomenon to advance in the development of models integrating this issue. With this aim, in a first phase, a test performance of different photoelastic models has been carried out, which has allowed establishing a set of different range solutions to the phenomenon models. In a second stage, these ranges were applied to the qualitative results of two types of numerical models: the first one is the simplest model possible within the method of the discrete elements (DEM), based on rigid blocks in unilateral contact; the second model is based on the method of linear finite elements (FEM) in which the contact surfaces have been modeled as voids, except for the one contact point. The results obtained using these qualitative comparisons provide new perspectives for the study of local behavior of masonry structures. A correct systematization of these combinations may be useful for the assessment of this type of masonry structures.
\end{abstract}

\section{INTRODUCTION}

\subsection{Photoelasticity applied to structural analysis}

Photoelasticity is a procedure developed in the early twentieth century to measure strain and stress in certain light-sensitive materials. Like those of any experimental procedure, photoelastic tests require a practical methodology (exceedingly simple: only a few cheap optical devices are needed, together with the photosensitive material model) but offers, in return, results of great interest. It allows the observer to easily establish stress patterns, identify areas of stress concentration or understand the general behavior of the studied element. From the 60 s of last century, the progressive development of numerical methods, supported by computational means, prevented its widespread use, although it has survived in some very specific areas.

Photoelastic analysis was used, and is still used today, in the study of the stress behavior of continuous media and, occasionally, of granular media. However, only seldom has it been used to study discontinuous orthotropic media, such as masonry elements. Some approaches, such as the one by R. Mark for the cross sections of Gothic cathedrals, used continuous models, ignoring the discontinuous condition of masonry and its internal organization, considerably orthotropic. The method described in the following pages aims to use photoelasticity in order to identify, at least qualitatively, the effect of the discontinuity of the material on the pattern of contact stresses caused by external actions. Constructive reality and experience in intervention in the existing heritage shows that, in masonry structures, the joints between blocks are often more important than the blocks themselves. Therefore, it is frequently not possible to anticipate their behavior numerically. An approach through the laws of randomness would allow knowing a given set of possible behaviors, but not necessarily the most likely.

\subsection{Photoelasticity applied to masonry. Background}

Although photoelastic tests have been frequent in the study of continuous media (Dally, 1991) its use in the analysis of discontinuous media has not. Studies on granular media (Drescher, 1972) often intended to evaluate the random nature of internal stress distribution, but this approach has only seldom been used in orthotropic media. The most systematic and interesting attempts are very recent (Bigoni 2010). However, there are some precedents. McNicholas (1970) used a birefringent coating for analysing tensions in a masonry model scale 1:1. Heinrich (1981) examined a voussoir arch, with strong friction in the joints, 
and associated internal stresses in the material with thrust lines and collapse mechanisms. Rajchenbach (2001) proposed a study of a model with rectangular blocks, similar to the one used here, analysing vertical distribution from a statistical perspective. Recently Bigoni (2010) proposed a model of rectangular blocks of with different photoelastic material, which were compared with mathematical models of random distribution. Finally Baig (2015), used a similar geometry, designed and graphic alteration algorithm to transform the photoelastic information into stress lines or streams.

\subsection{Objectives. Comparison of methods of analysis}

The aim of this study is to determine the relevance and interest of photoelastic tests, comparing their results with the most common numerical analysis methods in order to know the degree of approximation between them. Two numerical methods will be used for this comparison: the analysis of rigid blocks with random contact conditions and the finite element method.

\section{PHOTOELASTIC RESIN BLOCK MODEL TESTS}

The methodology used in this first set of tests is the usual in photoelastic analysis, but taken to the study of stress distribution in discontinuous media: in this case, a masonry wall with no mortar in the joints.

\subsection{Model. Materials and test conditions}

The physical model is as similar as possible both to the one used in the reference tests and to those mathematical models described in the paragraphs that follow. It is essentially a model that consists of a set of solid resin blocks arranged in direct contact. The material used is an epoxy resin, two components, polymerized at room temperature. The base is a commercial variant of epichlorohydrin; the curing agent, a monomer, BPA type. The resulting cured resin is a clear product with yellowish hues, for which the following compressive, tensile and flexural resistance can be estimated: 580,460 and $570 \mathrm{~kg} / \mathrm{cm}^{2}$ respectively. The material is mixed and casted into glass molds, producing flat plates of half a centimeter thick. These plates are cut with a standard laser cutter, combining high power ( $150 \mathrm{~W}$, so that the beam passes through the plate completely), with a medium-low speed of $\operatorname{arm}(0.01 \mathrm{~m} / \mathrm{s}$, to avoid burning resin).

The different blocks were installed in test position within a confinement PMMA box. The box was designed as a set of plates and fences to allow testing models up to $200 \times 300 \mathrm{~mm}$, in vertical and horizontal positions. In the front and back of the box two polarizing filters (polyvinyl alcohol iodin without color distortion and high efficiency of polarization -a cross transmission of 0.002 at $570 \mathrm{~nm}$ can be estimated)

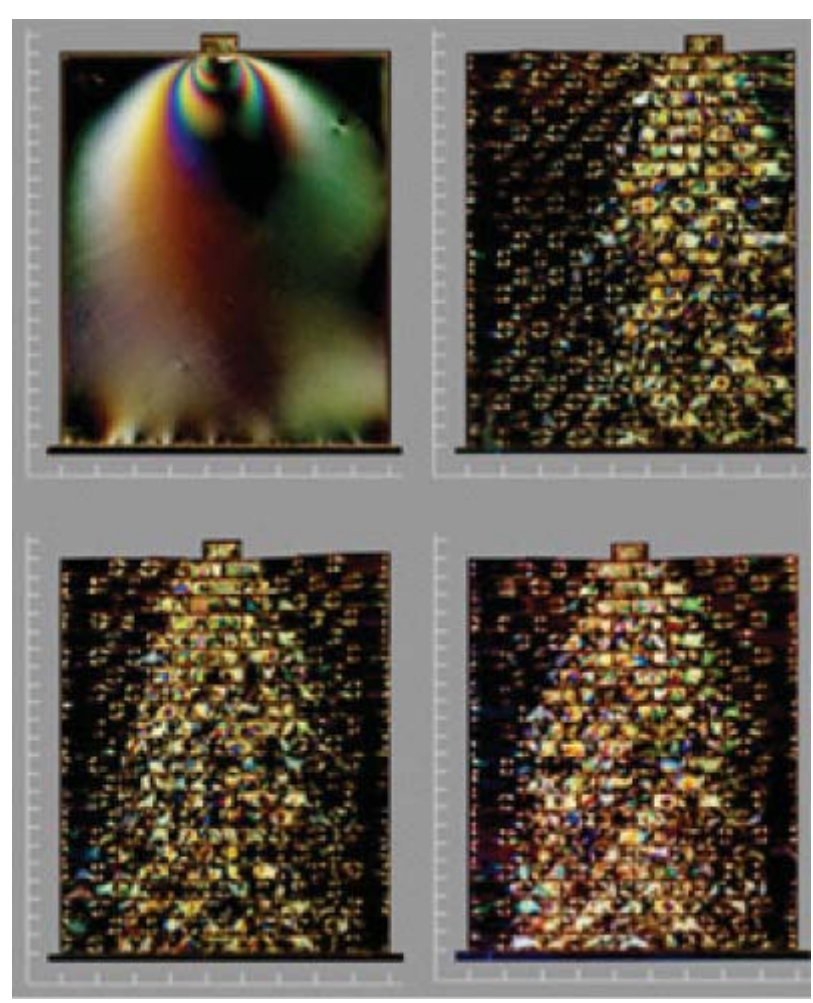

Figure 1a. Photoelastic tests. Results obtained in the test of an epoxy resin continuous model. Figures $1 b, c$, d. Photoelastic tests. Three of the results obtained in variation of the of the epoxy resin block models.

were added, making the whole installation a polariscope, one specifically designed for compression tests of small scale models.

\subsection{Implementation}

Different photoelastic tests were made. In all, several epoxy resin models were tested in compression within the confinement box described above. Compression was exerted by conventional mechanical means, using various PMMA pieces and a small block of resin, $20 \times 10 \times 10 \mathrm{~mm}$, in the contact. These tests were not to be quantitative, but qualitative (i.e. they are intended to show the range of stress distribution, not of its intensity) as the absolute values of loads and internal stresses are not relevant. However, one can estimate a maximum Q of $100 \mathrm{~N}$, a maximum stress of $0.5 \mathrm{~N} / \mathrm{mm}^{2}$ and an average stress at the base of $0.09 \mathrm{~N} / \mathrm{mm}^{2}$.

The first test (Fig. 1a) was performed on a single, continuous piece of $180 \times 220 \times 10 \mathrm{~mm}$. The intent of this initial approach was to confirm the theoretical properties of material, given that, in those products obtained by casting, internal irregularities could distort, to some extent, the results of the test. The result, as shown, is set to standard parameters for the resins used in the study of continuous media: the pressure bulb that shows the effect of the compression exerted is reasonably symmetrical; isochromatic lines consistent with resistant material values can be observed; and the deformation obtained is consistent with the expected elasticity module. 


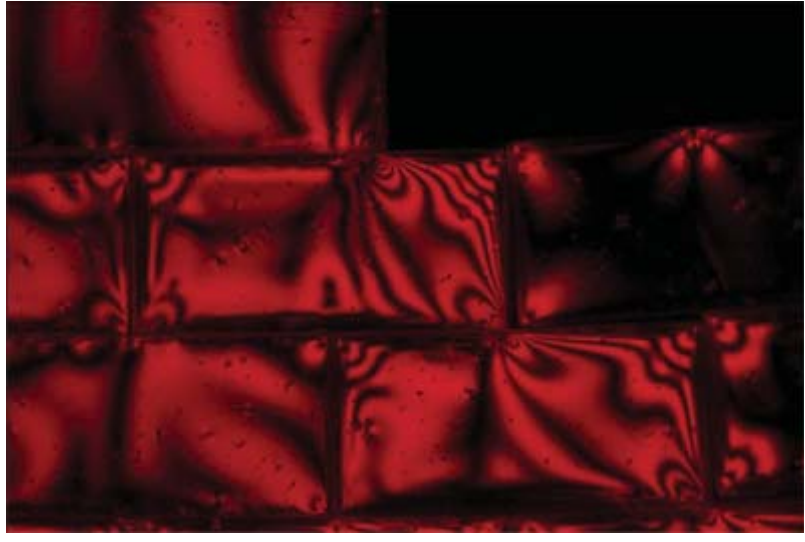

Figure 2. A closer view at the resin blocks in a stress flow area shows the concentration of stresses in specific edges and blocks.

Subsequent tests (Figs. 1b, 1c and 1d) were performed on a model that replicates the geometry of a masonry wall built with a conventional bond, in an identical distribution to that of the mathematical models that are detailed in the following paragraphs. For this purpose small blocks of $20 \times 10 \times 10 \mathrm{~mm}$ and $30 \times 10 \times 10$, arranged in an array of 180 wide by $220 \mathrm{~mm}$ high, were used. It took 187 of these blocks; 14 such tests, randomly rearranging the 187 blocks, were made.

\subsection{Results}

The first model (Fig. 1a) allows noticing that the interface between the blocks is imperfect, resulting in a random distribution of the points of application of contact forces. This is remarkable in the isochromatic lines that appear: in the left area up to five isochromatic bulbs can be noticed, while barely four are manifested under the right side of the model.

This irregularity is evident in the wide areas without stress and in the stress flows that could be appreciated in the block models. It can be noticed, comparing the accompanying figures, not only the existence of these uncharged areas, clearly visible (Figs 1b, c, d) but their variability, in size and position, during the experiments. This can also be said about the degree of dispersion of stress flows, extremely concentrated in specific points due to randomness in the sequence of contacts. Such irregularities in the distribution of stresses can have a significant effect on the local behavior of the wall (Fig. 2).

\section{MODEL OF RIGID BLOCKS WITH RANDOM CONTACT CONDITIONS}

The proposed method is included in those that Roca, Cervera \& Gariup (2010), in their recent review of methods applicable to the study of the historical masonry buildings, classified as "Advanced Computer Developments Based on Limit Analysis: Analysis of Blocky Structures".

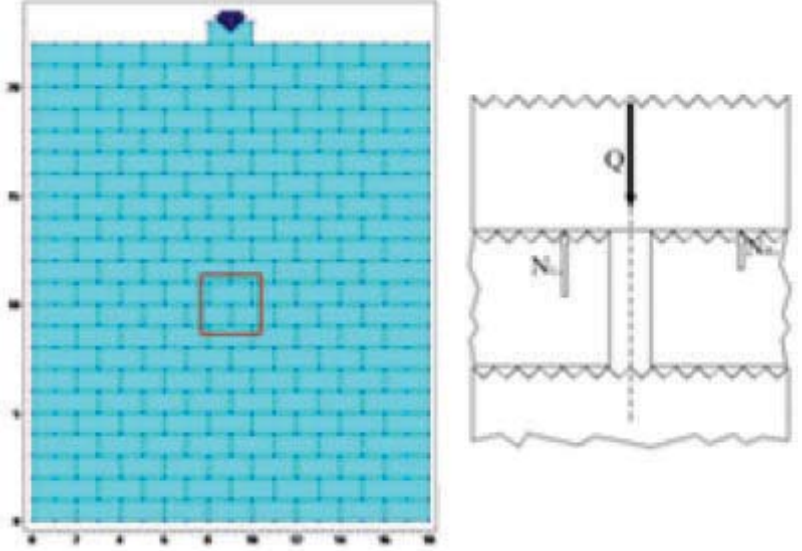

Figure 3. The model and its staple: random contact at the interfaces.

\subsection{Model}

A model composed of an assembly of rigid blocks in unilateral, dry and direct contact with finite friction (Fig. 3) is used. It is assumed that the contact surface between the blocks is imperfect and this causes a random distribution of the points of application of the contact forces.

Such models ignore the deformability of the material and its impact on the stress distribution, and highlights its discontinuous nature. It therefore tries to represent a material with a compressive strength much higher than the compressions to which it is subject, and little or no tensile strength.

Not considering the contribution of the tensile strength of the joint material is on the side of safety. A model of this type is suitable for a simplified analysis of historical masonry constructions, for which we usually do not have a record of damages over time and, in some cases, we do not even have many certainties about the current state of this material.

\subsection{Implementation}

Given the elemental nature of the problem studied, its implementation is simple and consists of the following steps:

1) The equilibrium equations for each of the blocks are set, taking into account that contact is unilateral and only compressive stresses in the contact surfaces are permitted.

2) Points of application of the contact forces are randomly selected using a uniform distribution. A different distribution would have been possible, but given the material stiffness this one is considered the most appropriate.

3) A feasible solution to balance the external force applied is sought using linear programming.

4) If a feasible solution is not found, because the added contact conditions have turned the problem overdetermined, it is rejected.

5) If it is found, this will be one of the possible solutions sought. 


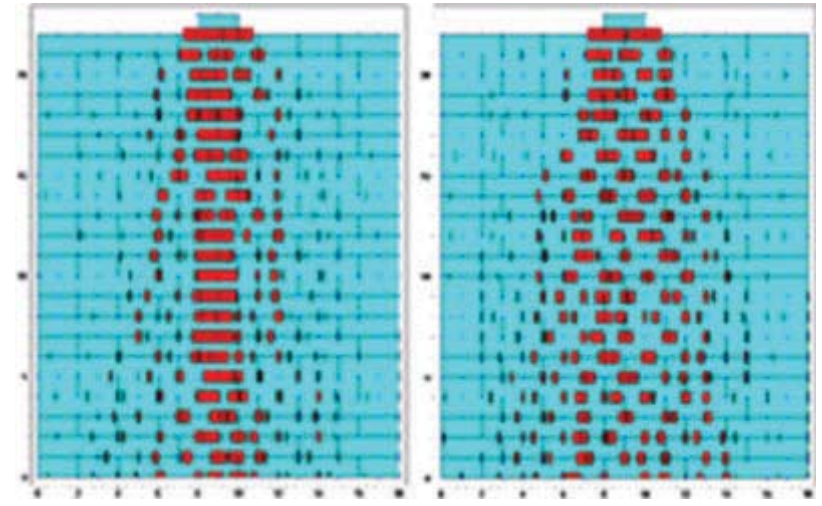

Figure 4. Results obtained in two of the cases studied.

\subsection{Results}

Since it is not intended to make a numerical comparison with the other methods, but only to make a comparison on a qualitative level, (Fig. 4) two distinct cases are included below. The strokes in red, in proportion to its width, represent the contact stresses.

It can be noticed that due to randomness in the contacts, the degree of dispersion of the contact stresses across the width of the model is very variable. Note that this irregularity in the distribution of stresses can have a significant effect on the local behavior of the wall.

\section{ANALYSIS BY FINITE ELEMENT METHOD}

The objective of the analysis described below is to use the finite element method to determine vertical stresses caused by an external load, and thus enable to locate the path of loads inside a masonry Wall. To achieve this, a finite element method analysis, SAP2000 (2009) was used. In order to compare the results of these analysis with the ones in the examples described above, the objective is to determine, using this methodology, the "way" that loads take inside the masonry instead of specifying the stresses of each of the blocks. It is not the intention of this analysis to describe deformations either, so the characterization of the material is not decisive, as it is in any qualitative analysis.

\subsection{Models}

\subsubsection{Continuous model (MC)}

The continuous model analyzed, called MC1, is configured by a mesh of $71 \times 87$ elements type "Shell Thin" of constant thickness, $10 \mathrm{~mm}$, and $2.5 \times 2.5 \mathrm{~mm}$ dimensions. The wall studied has therefore an overall dimension of $177.5 \mathrm{~mm}$ wide by $217.5 \mathrm{~mm}$ high. At the top, to simulate the application of a continuous load, the wall is topped with an addition of $17.5 \times 10.0 \mathrm{~mm}$, with the same mesh of the rest of the model. This is called by Baraldi, Cecchi \& Tralli (2015) a "flat continuous model".

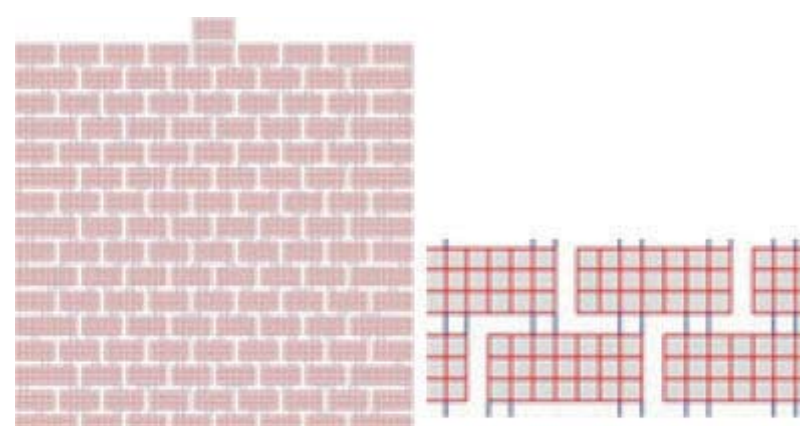

Figure 5. Global and local geometric description of the models analyzed (MDA).

\subsubsection{Discontinuous model with vertical joints (MJ)}

With the same overall dimensions and pieces of the previous example, in this case a space between the vertical joints has been included, with the standard grid step of $2.5 \mathrm{~mm}$. To model the continuous contact through the joint, and with the aim to understand the qualitative stress behavior inside the pieces representing the bricks, a series of bars have been placed as trusses. These trusses are called "frame" type by SAP2000, of infinite stiffness (compared to the rest of the model) and length of $2.5 \mathrm{~mm}$, connecting the lower nodes of the mesh with superior ones. To allow axial transmission exclusively, the transmission of other stresses between ends of the bars has been prevented.

\subsubsection{Discontinuous model without joints (MD)}

This example differs from the previous model. Here, the horizontal axial transmission is allowed through modeling the vertical joint the same way as the horizontal MJ model; This is, through adding horizontal trusses to connect nodes between brick blocks at the same high position.

\subsubsection{Model with random discontinuous support (MDA)}

For the discontinuous model pattern the same grid, the same block sizes and the same overall dimensions were used. To be able to compare with models in random contact, this condition has been introduced through a single contact between the possible nodes (Fig. 5)

Therefore the bars are placed vertically in a random contact pattern, so that, among the three blocks that may be in contact, only one is, by the two vertical bars connecting with the lower block. This model is called by Baraldi, Cecchi \& Tralli (2015) as heterogeneous finite element model (FEM Straight) adding the particularity of random contact between pieces.

\subsubsection{Material and constraints}

For the definition of the material the following parameters were established, taken obtained from Narayanan \& Sirajuddin (2013), as shown in Table 1. 
Table 1. Characterization of the material brick for analysis.

\begin{tabular}{ll}
\hline Property & Value \\
\hline Specific weight & $15 \mathrm{kN} / \mathrm{m}^{3}$ \\
Compressive strength & $4 \mathrm{~N} / \mathrm{mm}^{2}$ \\
Tensile strength & $0.4 \mathrm{~N} / \mathrm{mm}^{2}$ \\
Elastic modulus & $4.000 \mathrm{~N} / \mathrm{mm}^{2}$ \\
Poisson's ratio & 0,25 \\
\hline
\end{tabular}

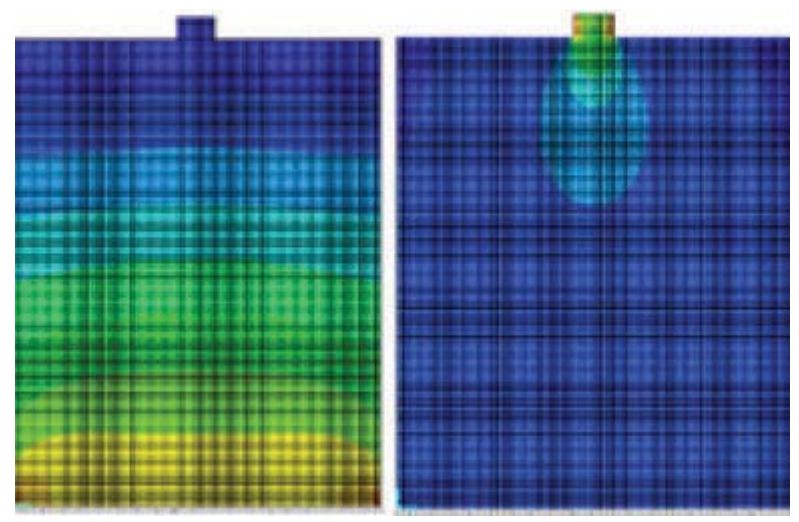

Figure 6. Results of the MC Analysis: Vertical Stresses (self weigh, left/ external load, right)

However, as mentioned above, the aim of this analysis is not to quantify the stress but to determine the stress flows, so the values shown in Table 1 can be varied without substantially modifying the results, except for the ratio weight - external load, which should be pondered proportionally. In all models the constraints are located at the corners of the bottom row and the supports are rigid, all movements and turns being thwarted. The tested models have not been restricted.

\subsubsection{Loads and load combinations. Analysis}

The load has a value of $1 \mathrm{~N}$ in the negative direction of $Z$ axis, in each of the 8 vertices of the upper element. In order to be able to clearly distinguish the tensions arising from the weight of the masonry and external loads, stresses are displayed with a combination of actions in a scale 250: 1 between the external loads and weight loads. A plane analysis is performed, cancelling forces along the $\mathrm{Y}$ axis and rotations around the axes $\mathrm{X}$ and Z. S22 stresses are obtained, corresponding to the normal stress in Z-direction.

\subsection{Results}

As in previous cases, the intention here is to make a qualitative analysis. Therefore numerical stress values are not included; only diagrams to enable an approach to the behavior of the material. To make it clear and produce greater visual contrast in quantifying stresses, a certain range is shown graphically, in order to highlight the stresses along the vertical axis of the masonry and to reduce the importance of stress concentration under

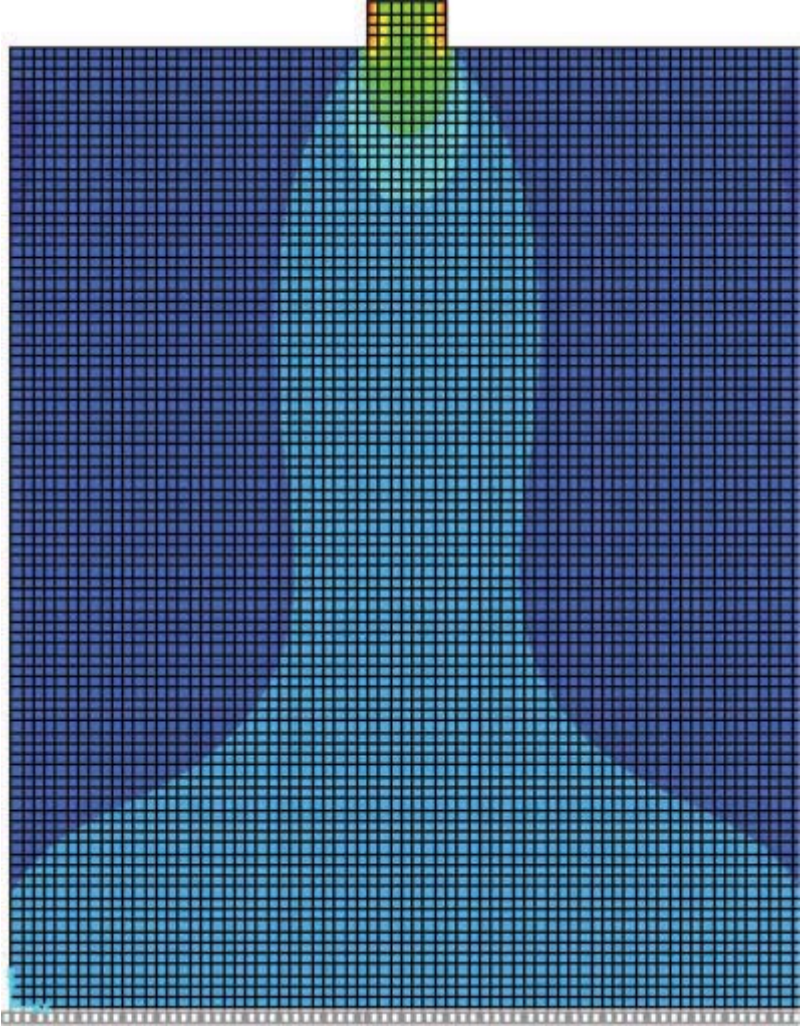

Figure 7. Results of the MC Analysis in Combination of Actions.
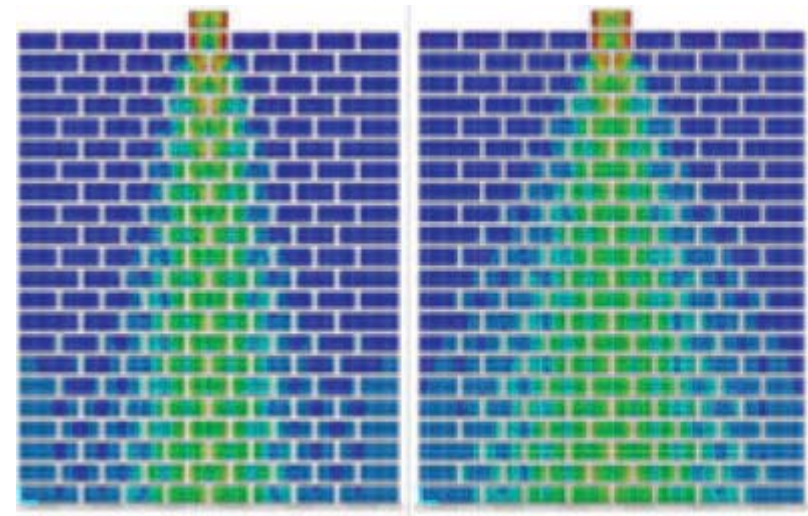

Figure 8. Results of the MJ (left) and MD (right) Analysis: Vertical Stresses in Combination of Actions.

the application points, displaying a range of tensions between $-0.20 \mathrm{~N} / \mathrm{mm}^{2}$ and $0 \mathrm{~N} / \mathrm{mm}^{2}$ in all models.

Figures $6 \mathrm{a}, \mathrm{b}$ explain vertical stress both simple loads as combination of them. Figure 7 shows the results of analysis for the MC under their combination. The result of the combination of external load and self weight for MJ model is shown in Figure 8a and MD in Figure 8b. Finally, the vertical stresses for four random contacts.

\section{DISCUSSION OF RESULTS}

The comparison between the results of these three methods, and between them and the reference tests 

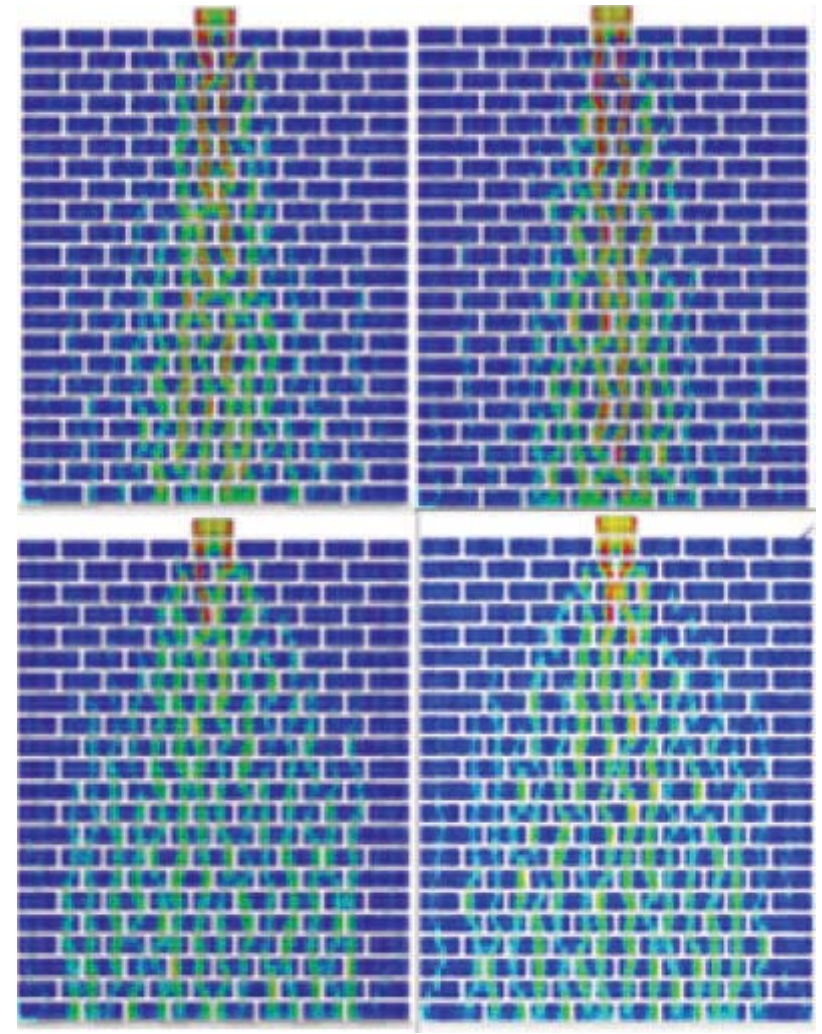

Figure 9. Results of the analysis of different MDA models: Vertical Stresses in Combination of Actions.

(Bigoni \& Noselli, 2010) will allow establishing some interesting conclusions. Before that, however, a few clarifications are in order.

Regarding the photoelastic tests included in this communication, it should be noted that these were performed with a single material, an epoxy resin, unlike those undertaken by Bigoni and Noselli, performed using different photosensitive materials. The results obtained in our tests are consistent with the photoelastic reference records; however, by using a single material our responses range is more limited. The intent of these tests has always been, in any case, to compare these photoelastic results, of indicial character, not with others of the same type, but with different mathematical models.

Also note that the resin used is a resilient material, which may, to some extent, distort results. Thus, the opening angle obtained between the surface of application of the force and the reaction on the base is somewhat bigger, in most cases, than the ones obtained in the different mathematical models, which may be attributable to the greater rigidity of the latter.

\section{CONCLUSIONS}

The overall performance of a masonry structure does not differ significantly when analyzed as a continuous or a discontinuous medium. However, great differences can be observed at a local level when one or other medium is considered, especially when taking into account the unknown contacts between pieces, an effect that does occur quite often in historical masonry elements. In case local problems were detected in a real masonry structure -problems due to, say, damages in the bonding material-these methods could easily be implemented, allowing to detect to what extent, in such case, the adherence resistance depends on friction. The appearance of islets of very low or no tension inside the masonry has great relevance in the local behavior, going unnoticed when analyzed globally.

The photoelastic method has proven useful to visualize the stress flows inside constructive elements, as it allows understanding the qualitative behavior of masonry structures.

\section{ACKNOWLEDGMENTS}

The first author would like to thank the Juanelo Turriano Foundation for supporting this work through a scholarship for the realization of his doctoral dissertation.

\section{REFERENCES}

Baig, I., Ramesh, K. \& Hariprasad, M. P. 2015. Analysis of stress distribution in dry masonry walls using three fringe photoelasticity. International Conference on Experimental Mechanics 2014. Cambridge: International Society for Optics and Photonic. 93022P-93022P.

Baraldi, D., Cecchi, A. \& Tralli, A. 2015. Continuous and discrete models for masonry like material: A critical comparative study. European Journal of Mechanics A/Solids 50: 39-58.

Bigoni, D. \& Noselli, G. 2010. Localized stress percolation through dry masonry walls. Part I - Experiments \& Part II - Modelling European Journal of Mechanics A/Solids, 29, 291-298 \& 299-307.

Dally, J. W. \& Riley, W. F. 1991. Experimental stress analysis. New York: McGraw-Hill.

Drescher, A., de Josseling de Jong, G. 1972. Photoelastic verification of a mechanical model for the flow of a granular material. Journal of the Mechanics and Physics of Solids, 20(5): 337-340.

Heinrich, B. 1977. Am Anfang war der Balken. Technik lernen mit übergreifenden Bezügen. Kultur\& Technik 1: 38-45.

McNicholas, J. B. 1970. Photoelastic Stress Analysis of Brick Masonry Systems. 2nd International Brick and Block Masonry Conference.: Stoke-on-Trent: British Masonry Society. 88-94.

Narayanan, S. P. \& Sirajuddin, M. 2013. Properties of Brick Masonry for FE modeling. Recent Avances in Structural Engineering RASE2013. American Journal of Engineering Research (AJER). 1, 06-11.

Roca, P., Cervera, M. \& Gariup, G. 2010. Structural analysis of masonry historical constructions. Classical and advanced approaches. Archives of Computational Methods in Engineering, 17(3), 299-325.

Rajchenbach, J. 2001. Stress transmission through a cohesionless material. Material Physics Mechanichs 3: 1-4.

SAP2000 Advanced 14.0.0. 2009. Structural Analysis Software. Computers and Structures, Inc. 